\title{
Lattice Boltzmann Model for Simulating Immiscible Two-Phase Flows
}

\author{
T. Reis and T. N. Phillips \\ School of Mathematics, Cardiff University, Cardiff, CF24 4AG, United Kingdom \\ E-mail: ReisT@cf.ac.uk, PhillipsTN@cf.ac.uk
}

\begin{abstract}
The lattice Boltzmann equation is often promoted as a numerical simulation tool that is particularly suitable for predicting the flow of complex fluids. This article develops a two-dimensional 9 velocity (D2Q9) lattice Boltzmann model for immiscible binary fluids with variable viscosities and density ratio using a single relaxation time for each fluid. In the macroscopic limit this model is shown to recover the Navier-Stokes equations for two-phase flows. This is achieved by constructing a two-phase component of the collision operator that induces the appropriate surface tension term in the macroscopic equations. A theoretical expression for surface tension is determined. The validity of this analysis is confirmed by comparing numerical and theoretical predictions of surface tension as a function of density. The model is also shown to predict Laplace's law for surface tension and Poiseuille flow of layered immiscible binary fluids. The spinodal decomposition of two fluids of equal density but different viscosity is then studied. At equilibrium, the system comprises one large low viscosity bubble enclosed by the more viscous fluid in agreement with theoretical arguments of Renardy and Joseph [1]. Two other simulations, namely the nonequilibrium rod rest and the coalescence of two bubbles, are performed to show that this model can be used to simulate two fluids with a large density ratio.
\end{abstract}

AMS classification scheme numbers: 76M28,76D99,76D05

Submitted to: J. Phys. A: Math. Gen. 


\section{Introduction}

The numerical simulation of multiphase flow problems requires some procedure for tracking the interface(s) between the different phases as they evolve in time. Conventional continuum-based numerical methods are not always suitable for these sort of calculations. For example, a Lagrangian approach can accurately track an interface by attaching to it a number of probes, the dynamics of which follow the boundary evolution. However, if the interface topology is radically altered this method can suffer from illconditioning and singularities, and, due to the necessity to mesh complex geometries, three-dimensional computations can prove to be costly. An Eulerian approach on the other hand can overcome these difficulties since large deformations in an interface can be captured without a re-discretisation of the domain. Rather than track the interface explicitly, this method reconstructs it as an isocontour of a field variable. The problem with this technique is, due to the lack of explicit treatment, interfacial diffusion effects are generally smeared over a region surrounding the interface.

In the classical continuum approach the equations of motion that hold in each fluid are solved with appropriate conditions defined at the interface between the fluids. The interface is a free surface that evolves in time and the conditions that hold there involve the physical properties of the fluids such as surface tension. An alternative description of immiscible two-phase flows is based on diffuse-interface models in which quantities such as surface tension, for example, are distributed throughout an interfacial region. In such a description, surface tension is represented as a distributed stress within this region.

From a micro/mesoscopic view point the segregation of two fluids is due to interparticle forces. The Lattice Boltzmann method is therefore in a strong position compared to its macroscopic rivals since these particle interactions can be incorporated into the evolution of the distribution function. As a result, a multi-phase Lattice Boltzmann model should not track interfaces but rather let them emerge spontaneously from the underlying dynamics.

A number of Lattice Boltzmann models have been developed to predict the flow of two interacting fluids, each showing a degree of success in a variety of test situations but also several limitations.

Rothman and Keller [2] were the first to propose an extension to the single phase lattice gas model of Frisch et al [3] (the so-called FHP model) to multiphase problems. In the multiphase FHP model of Rothman and Keller [2] coloured particles are introduced to distinguish between the phases. Furthermore, a nearest neighbour particle interaction is used to facilitate interfacial dynamics such as Laplace's formula for surface tension. The latter is accomplished by modifying the collision process to encourage 'like' particles to congregate.

The original coloured particle scheme of Rothman and Keller [2] was extended by Somers and Rem [4] and Chen et al. [5] by introducing coloured holes. Chen et al. [5] showed that this approach resulted in an extension of the nearest neighbour particle 
interaction to several lattice lengths. Furthermore, since this scheme carries purely local information in its particle collision step, the size of the look-up table in the algorithm is reduced and consequently there is an improvement in the efficiency of the algorithm.

Although multiphase lattice gas algorithms are able to produce interesting surface phenomena it is difficult to make quantitative comparison with experiments and other numerical simulation techniques due to noise induced by particle fluctuations.

The lattice Boltzmann model (LBM) of McNamara and Zanetti [6] solves the kinetic equation for the particle distribution instead of tracking the motion of each particle and is therefore less susceptible to noise. Since lattice gas algorithms contain more information about the microscopic behaviour of particles they will be superior to LBM for simulating the underlying physics and modelling microscopic dynamics such as correlation effects and phase transitions. However, LBM's come into their own as a numerical tool for solving PDEs governing macroscopic behaviour. As with lattice gas algorithms LBM can be efficiently implemented on parallel computers and boundary conditions can be treated easily. Boundary conditions on curved boundaries can be treated using interpolation methods to calculate the distribution functions on the boundary. For example, Yu et al. [7] presented a second-order treatment of boundary conditions on curved boundaries.

The pseudo-potential approach introduced by Shan and Chen [5] is a multi-phase LBM that attempts to be a more physically orientated model than the R-K models. Since flows with more than one phase have a non-ideal equation of state, Shan and Chen [5] looked to preserve this feature in a lattice Boltzmann framework by incorporating non-local interactions among particles. Chin et al. [8] used a Shan-Chen model on a D2Q9 lattice with the LBGK collision operator to simulate flows of immiscible fluids with different viscosities. Their prediction of Laplace's law for surface tension gives noticeable errors and their simulated results for Poiseuille flow, although in close agreement in single fluid regions, shows discrepancies near an interface.

Due to its simplicity and elegance the Shan-Chen model is probably the most popular choice for flows with phase transitions. Encouraging results in comparison to thermodynamic theory have been obtained $[5,9,10]$. There are unfortunately a number of drawbacks. As pointed out by Nourgaliev et al. [11] this model cannot introduce a temperature which is consistent with thermodynamics and Luo [12] shows that the equation of state is not the same in the momentum equation and the energy equation.

Striving to ensure thermodynamic consistency within the lattice Boltzmann framework, Swift et al. [13] and Orlandini et al. [14] introduced phase effects directly into collision process by considering a generalised equilibrium function that includes non-ideal pressure tensor terms. These terms are defined to cohere with the free-energy functional in diffuse interface theory. To test this model Swift et al. [13] performed numerical simulations (on a FHP lattice with one rest particle) using a Van-de-Waals equation of state. They report a very good agreement between the mechanical (Laplace's law) and thermodynamic definitions of surface tension. Also shown in [13] is the accurate prediction of the coexistence curve between the two phases for different fluid 
temperatures. Further work with the free energy model was undertaken by Inamuro, Nobuharu and Ogino [15] who showed Galilean invariance of the model in moving droplet deformation and breakup simulations, and $\mathrm{Xu}$, Gonnella and Lamura [16] improved the stability of the model by controlling density fluctuations.

Theoretical criticisms of the free energy approach are made by Luo $[12,17]$. He points out that since density gradients do not appear in the first order Chapman-Enskog expansion the inclusion of density gradient terms is not justified. An in-depth study reveals other shortcomings such as a degree of anisotropy and varying temperature even though the model claims to be isothermal. Although all the algorithms considered so far in this section are in some way mathematically ad hoc it appears from the work of Luo that the free energy model is also physically incorrect.

Gunstensen et al. [18] proposed a LBM for multiphase flows that combined the single-phase LBM of McNamara and Zanetti [6] with the multiphase lattice gas algorithm of Keller and Rothman [2]. An important contribution in this article is the introduction of a perturbation step in order to recover Laplace's formula at an interface. This is achieved by adding a binary fluid collision operator to the post collision state at sites near the interface. The role of this operator is to deplete mass along lattice links parallel to an interface and add mass to lattice links perpendicular to an interface, while conserving the total mass and momentum at the site. Although Galilean invariance is recovered by the proper assignment of rest particles, the model does not solve the exact governing equations for multiphase flow. Furthermore, the model uses a fully linearized collision operator which is computationally inefficient in 3D and it is restricted to flows in which the fluids have the same densities and viscosities.

A similar model that allows for different densities and viscosities was proposed by Grunau et al. [19] by the incorporation of the freedom of the rest particle equilibrium distribution function and a space dependent relaxation process. The incompressible Navier-Stokes equations are also recovered for the colour-blind fluid. The advantage of models based on the Rothman-Keller model [19, 18] is that surface tension, the density ratio and the viscosity ratio can be chosen independently. Tölke et al. [20] proposed another adaptation of the Rothman-Keller type model introduced by Gunstensen et al. [18] based on unstructured tree-type grids in which an additional lattice Boltzmann equation is used to advect the phase field. However, a recolouring step is still required to eliminate diffusion effects and to maintain a sharp interface.

In this paper we propose an immiscible lattice Boltzmann model for binary fluids that is similar to the model of Gunstensen et al. [18] but with several important modifications. First, the two-phase operator is adjusted and shown to recover the single phase Navier-Stokes equations, with an appropriate source term to model surface tension, in the macroscopic limit. As a result, a new theoretical expression for the surface tension coefficient is found from the analysis of the model. Furthermore, extensive numerical experiments show this model is capable of predicting flows with large density ratios unlike the original model of 


\section{Immiscible Lattice Boltzmann Model}

We develop a R-K-type model for immiscible binary fluids with different densities and viscosities, similar to the work of Grunau et al [19]. Like these authors we use a lattice Boltzmann equation with a single relaxation parameter for each fluid but use a D2Q9 lattice rather than a 7-velocity FHP lattice with additional rest particles. The equilibrium expressions given in this section, found by a simple ansatz, thus differ to those in [19] and the collision operator is modified to satisfy the conservation laws and recover the extra volume source term in the multi-phase Navier-Stokes equations.

Let $N_{i}^{k}$ be the single particle distribution function for fluid $k$, where $k=r$ or $b$ denotes the colour ('red' or 'blue'). The total population at node $\mathbf{x}$ and time $t$ is

$$
N_{i}(\mathbf{x}, t)=N_{i}^{r}(\mathbf{x}, t)+N_{i}^{b}(\mathbf{x}, t),
$$

and the evolution equation for each phase is

$$
N_{i}^{k}\left(\mathbf{x}+\mathbf{c}_{i}, t+1\right)=N_{i}^{k}(\mathbf{x}, t)+\Omega_{i}^{k}(\mathbf{x}, t) .
$$

The collision operator

$$
\Omega_{i}^{k}=\left(\Omega_{i}^{k}\right)^{(1)}+\left(\Omega_{i}^{k}\right)^{(2)}, \quad i=0, \ldots, 8
$$

consists of two processes. The first represents relaxation to a local equilibrium state using, for simplicity, an LBGK operator:

$$
\left(\Omega_{i}^{k}\right)^{(1)}=-\omega_{k}\left(N_{i}^{k}-N_{i}^{k(e)}\right),
$$

where $N_{i}^{k(e)}$ is an equilibrium function and $\omega_{k}$ is the relaxation parameter of fluid $k$. The operator $\left(\Omega_{i}^{k}\right)^{(2)}$ is the two-phase component of the collision operator and is derived in Section 2.1.

Mass and momentum are, as usual, defined to be the first two moments of the distribution function respectively, and conservation of these quantities require

$$
\begin{aligned}
\rho_{k} & =\sum_{i} N_{i}^{k}=\sum_{i} N_{i}^{k(e)} \\
\rho \mathbf{u} & =\sum_{i} \sum_{k} N_{i}^{k} \mathbf{c}_{i}=\sum_{i} \sum_{k} N_{i}^{k(e)} \mathbf{c}_{i},
\end{aligned}
$$

where $\rho_{k}$ is the density of fluid $k, \rho=\rho_{r}+\rho_{b}$ is the total density, and $\mathbf{u}$ is the local fluid velocity.

The equilibrium function $N_{i}^{k(e)}$ can be chosen arbitrarily providing it respects the conservation constraints of equations (5) and (6). To derive an expression we specify a D2Q9 lattice domain and use the following ansatz:

$$
\begin{aligned}
& N_{0}^{k(e)}=A_{0}^{k}+D_{0}^{k} \mathbf{u}^{2}, \\
& N_{i}^{k(e)}=A_{1}^{k}+B_{1}^{k} \mathbf{c}_{i} \cdot \mathbf{u}+C_{1}^{k}\left(\mathbf{c}_{i} \cdot \mathbf{u}\right)^{2}+D_{1}^{k} \mathbf{u}^{2}, \\
& \quad \text { for } i=1,2,3,4, \\
& N_{i}^{k(e)}=A_{2}^{k}+B_{2}^{k} \mathbf{c}_{i} \cdot \mathbf{u}+C_{2}^{k}\left(\mathbf{c}_{i} \cdot \mathbf{u}\right)^{2}+D_{2}^{k} \mathbf{u}^{2}, \\
& \quad \text { for } i=5,6,7,8,
\end{aligned}
$$


where the 10 capital letters are free parameters to be 'tuned' accordingly. To achieve a stable interface we require the non-stationary distribution functions for both liquids to be equal when $\mathbf{u}=0$. To meet this condition we assume

$$
\frac{B_{1}^{k}}{B_{2}^{k}}=\frac{D_{0}^{k}}{D_{1}^{k}}=\frac{D_{1}^{k}}{D_{2}^{k}}=r,
$$

where $r$ is some constant. Now assume

$$
\begin{aligned}
& A_{0}^{k}=\alpha_{k} \rho_{k}, \\
& \frac{A_{1}^{k}}{A_{2}^{k}}=r,
\end{aligned}
$$

where $\alpha_{k}$ is a free parameter. By taking advantage of the LBGK moments and respecting isotropy the equilibrium functions are readily found to be

$$
\begin{aligned}
N_{0}^{k(e)}= & \rho_{k}\left(\alpha_{k}-\frac{2}{3} \mathbf{u}^{2}\right) \\
N_{i}^{k(e)}= & \rho_{k}\left(\frac{1-\alpha_{k}}{5}+W_{i}\left[3 \mathbf{c}_{i} \cdot \mathbf{u}+\frac{9}{2}\left(\mathbf{c}_{i} \cdot \mathbf{u}\right)^{2}-\frac{3}{2} \mathbf{u}^{2}\right]\right), \\
& \quad i=1,2,3,4, \\
N_{i}^{k(e)}= & \rho_{k}\left(\frac{1-\alpha_{k}}{20}+W_{i}\left[3 \mathbf{c}_{i} \cdot \mathbf{u}+\frac{9}{2}\left(\mathbf{c}_{i} \cdot \mathbf{u}\right)^{2}-\frac{3}{2} \mathbf{u}^{2}\right]\right), \\
& i=5,6,7,8
\end{aligned}
$$

where the weights $W_{i}$ are given by

$$
W_{i}=\left\{\begin{aligned}
\frac{4}{9}, & i=0, \\
\frac{1}{9}, & i=1,2,3,4, \\
\frac{1}{36}, & i=5,6,7,8 .
\end{aligned}\right.
$$

The stable interface assumption leads to the following density ratio, $\gamma$ :

$$
\gamma=\frac{\rho_{r}}{\rho_{b}}=\frac{1-\alpha_{b}}{1-\alpha_{r}}
$$

with the pressure given by:

$$
p_{0}^{k}=\frac{3 \rho_{k}\left(1-\alpha_{k}\right)}{5}=\rho_{k}\left(c_{s}^{k}\right)^{2}
$$

which satisfies an ideal equation of state. The parameter $\alpha_{k}$ determines the speed of sound, $\left(c_{s}^{k}\right)^{2}$, thus controlling the hydrodynamic pressure at interfaces. It can be viewed as representing the ensemble average number of degenerate rest particles, which is needed to achieve a stable interface and achieve a density variation between the fluids. Therefore the choice of $\alpha_{k}$ is important for flows with a large density difference. To ensure that $0<N_{i}^{k}<1$ in a mixed region we require

$$
0<\alpha_{k}<1
$$

and a little manipulation reveals that

if $\rho_{r}>\rho_{b}$.

$$
\frac{\rho_{r}-\rho_{b}}{\rho_{r}}<\alpha_{r}<1
$$




\subsection{Two-Phase Collision Operator}

The two-phase collision operator, $\left(\Omega_{i}^{k}\right)^{(2)}$, is defined in such a way as to encourage colour segregation while satisfying the conservation constraints of equations (5) and (6). A spatial colour difference, $\bar{\rho}$, is defined as

$$
\bar{\rho}(\mathbf{x})=\rho_{r}(\mathbf{x})-\rho_{b}(\mathbf{x}) .
$$

The colour gradient may be calculated in terms of the colour difference using

$$
\mathbf{H}(\mathbf{x})=\nabla \bar{\rho}(\mathbf{x}) .
$$

A fourth-order approximation, $\mathbf{F}$, to the colour gradient, $\mathbf{H}$, is given by

$$
\mathbf{F}(\mathbf{x})=\sum_{i=1}^{8} \mathbf{c}_{i}\left[\rho_{r}\left(\mathbf{x}+\mathbf{c}_{i}\right)-\rho_{b}\left(\mathbf{x}+\mathbf{c}_{i}\right)\right]
$$

Since the colour gradient is perpendicular to the interface, we can define an approximate unit normal, $\mathbf{n}$, to the surface:

$$
\mathbf{n}=\frac{\mathbf{F}}{|\mathbf{F}|} \simeq \frac{\nabla \bar{\rho}}{|\nabla \bar{\rho}|}
$$

An extension of the model proposed by Grunau et al. [19] to a D2Q9 lattice would give

$$
\left(\Omega_{i}^{k}\right)^{(2)}=\frac{A_{k}}{2}|\mathbf{F}|\left[\frac{\left(\mathbf{F} \cdot \mathbf{c}_{i}\right)^{2}}{|\mathbf{F}|^{2}}-\frac{3}{4}\right], \quad i=1, \ldots, 8 .
$$

In equation (23) $A_{k}$ is a free parameter controlling surface tension. We note that $\mathbf{F}=0$ in pure phases, thus $\left(\Omega_{i}^{k}\right)^{(2)}$ only contributes to mixed interfacial regions. The factor $3 / 4$ in equation (23) is included to ensure the conservation of mass and momentum:

$$
\begin{aligned}
& \sum_{i=1}^{8}\left(\Omega_{i}^{k}\right)^{(2)}=0 \\
& \sum_{i=1}^{8}\left(\Omega_{i}^{k}\right)^{(2)} \mathbf{c}_{i}=0 .
\end{aligned}
$$

However, it can be shown using the Chapman-Enskog technique that it is not possible to derive the correct form of the macroscopic continuum equations for multiphase flow when the two-phase collision operator is defined by (23). To obtain the correct form of the continuum equations we propose the following representation of the two-phase collision operator

$$
\left(\Omega_{i}^{k}\right)^{(2)}=\frac{A_{k}}{2}|\mathbf{F}|\left[W_{i} \frac{\left(\mathbf{c}_{i} \cdot \mathbf{F}\right)^{2}}{|\mathbf{F}|^{2}}-B_{i}\right], \quad i=0, \ldots, 8,
$$

where

$$
B_{i}=\left\{\begin{aligned}
-\frac{4}{27}, & & i=0 \\
\frac{2}{27}, & i & =1,2,3,4 \\
\frac{5}{108}, & i & =5,6,7,8 .
\end{aligned}\right.
$$


The colour gradient $\mathbf{F}(\mathbf{x})$ is normal to the interface at node $\mathbf{x}$ (see Fig. 1) so we see that $\left(\Omega_{i}^{k}\right)^{(2)}$ in $(23)$ serves to add mass to populations moving in this direction and removes mass parallel to the interface. Since this term does not conserve colour densities separately an additional step is needed to promote phase segregation and maintain surfaces between fluids. Following Gunstensen [18], we define the colour flux $\mathbf{K}(\mathbf{x})$ by

$$
\mathbf{K}(\mathbf{x})=\sum_{i=1}^{8}\left(N_{i}^{r}-N_{i}^{b}\right) \mathbf{c}_{i},
$$

and force this vector to align with the colour gradient (22) and minimise the diffusion of one colour into the other.

Figure 1. The colour gradient $\mathbf{F}$ is normal to a fluid-fluid interface.

2.1.1. Re-colouring The optimisation problem above can be written as follows: Maximise the work done $W$ against the colour gradient:

$$
W=\mathbf{K} \cdot \mathbf{F},
$$

subject to the constraints

$$
\begin{aligned}
N_{i}^{r \prime \prime}+N_{i}^{b \prime \prime} & =N_{i}^{\prime \prime}, \\
\sum_{i} N_{i}^{r \prime \prime} & =\rho_{r},
\end{aligned}
$$

where the double primes denote post two-phase collision quantities. Differentiating $W$ with respect to $N_{i}^{r \prime \prime}$ and $N_{i}^{b \prime \prime}$ yields

$$
\begin{aligned}
& \frac{\partial W}{\partial N_{i}^{r \prime \prime}}=\sum_{i}\left(\mathbf{c}_{i} \cdot \mathbf{F}\right)=0, \\
& \frac{\partial W}{\partial N_{i}^{b \prime \prime}}=-\sum_{i}\left(\mathbf{c}_{i} \cdot \mathbf{F}\right)=0,
\end{aligned}
$$

i.e. there are no turning points. Optimisation techniques such as the method of Lagrangian multipliers are therefore redundant. We continue to solve the maximisation problem in a more $a d h o c$ fashion. The link vectors $\mathbf{c}_{i}$ are listed in descending order starting with the one nearest the colour gradient $\mathbf{F}$. The maximum number of red 
particles available are sent in the directions close to $\mathbf{F}$ (i.e. perpendicular to the interface) while blue particles are sent in the opposite direction, subject to constraints (30) and (31). Latva-Kokko and Rothman [21] point out that a potential drawback of this re-colouring technique is so called lattice pinning. This situation occurs when one of the fluids, say red, is close to or on a fluid-fluid interface but the flow is too weak to move many red particles. The interface now becomes pinned to the lattice. Latva-Kokko and Rothamn studied this effect in the case of small bubbles concentrated around one lattice node. The authors report that such bubbles will not move unless forced very hard - a problem which can be of significance when examining the flow and separation of an initially mixed state. Alternative re-colouring schemes which reduce lattice pinning but widen the interface have been suggested by Latva-Kokko and Rothman [21] and Tölke et al. [20].

2.1.2. Interface relaxation parameter The thickness of an interface will depend on an averaged relaxation parameter. When the relaxation parameters $\omega_{k}$, and therefore the viscosities, of the two fluids are different, the interface width increases. To ensure a stable interface and smooth change in viscosity we define an order parameter $\psi$ in the same fashion of Grunau [19] et al.:

$$
\psi=\frac{\rho_{r}-\rho_{b}}{\rho_{r}+\rho_{b}}
$$

and a relaxation function $\omega \equiv \omega(\psi)$ is defined as follows:

$$
\omega= \begin{cases}\omega_{r}, & \psi>\delta \\ f_{r}(\psi), & \delta \geq \psi>0 \\ f_{b}(\psi), & 0 \geq \psi \geq-\delta, \\ \omega_{b}, & \psi<-\delta\end{cases}
$$

where

$$
\begin{aligned}
& f_{r}(\psi)=\beta+\gamma \psi+\epsilon \psi^{2}, \\
& f_{b}(\psi)=\beta+\eta \psi+\xi \psi^{2},
\end{aligned}
$$

and $\beta, \gamma, \epsilon, \eta$ and $\xi$ are constants chosen so that $\omega$ and its derivative are continuous. Let $\langle\omega\rangle=2 \omega_{r} \omega_{b} /\left(\omega_{r}+\omega_{b}\right)$ be the averaged relaxation parameter across the interface and assume that $f_{r}(\delta)=\omega_{r}, f_{b}(-\delta)=\omega_{b}, \partial \omega / \partial \psi=0$ when $\psi= \pm \delta$, and $f_{r}(0)=f_{b}(0)=\langle\omega\rangle$. Simple algebra reveals that

$$
\begin{aligned}
& \beta=\langle\omega\rangle, \\
& \gamma=\frac{2\left(\omega_{r}-\beta\right)}{\delta}, \\
& \epsilon=-\frac{\gamma}{2 \delta}, \\
& \eta=\frac{2\left(\beta-\omega_{b}\right)}{\delta}, \\
& \xi=\frac{\eta}{2 \delta}
\end{aligned}
$$


where $\delta \leq 1$ is a free parameter controlling the thickness of an interface. If the relaxation parameters (and therefore viscosities) are equal the value of $\delta$ does not affect the flow. If on the other hand there is a large difference in viscosity between the two fluids the choice of $\delta$ could affect the thickness and dynamics of an interface.

\subsection{Surface Tension}

Figure 2. Planar interface geometry in Cartesian co-ordinates.

The mechanical definition of surface tension is

$$
\sigma=\int_{-\infty}^{\infty}\left(P_{N}(z)-P_{T}(z)\right) d z
$$

where $P_{N}$ and $P_{T}$ are the normal and tangential components of the pressure tensor and $z$ measures the distance normal to the interface. Let $\theta_{i}$ be the angle between link vector $\mathbf{c}_{i}$ and the $x$-axis and let $\theta_{z}$ be the angle between $z$ and the $x$-axis (see Fig. 2). The components $P_{N}$ and $P_{T}$ are given by

$$
\begin{aligned}
& P_{N}=\sum_{i} N_{i} c_{i N}^{2}, \\
& P_{T}=\sum_{i} N_{i} c_{i T}^{2},
\end{aligned}
$$

where

$$
\begin{aligned}
& c_{i N}=\left|\mathbf{c}_{i}\right| \cos \left(\theta_{i}-\theta_{z}\right), \\
& c_{i T}=\left|\mathbf{c}_{i}\right| \sin \left(\theta_{i}-\theta_{z}\right) .
\end{aligned}
$$

Consider equation (43) as an average over $M$ adjacent long integration lines $y=$ constant and cast a discrete summation over lattice nodes in an area $A[22]$ :

$$
\sigma \approx \frac{\cos \theta_{z}}{M} \sum_{\mathbf{x} \in A} \sum_{i} N_{i} U_{i}=\frac{\cos \theta_{z}}{M} \sum_{\mathbf{x} \in A} \sum_{i}\left(N_{i}^{(e)}+N_{i}^{(n e q)}\right) U_{i},
$$

where

$$
U_{i}=\mathbf{c}_{i}^{2} \cos \left[2\left(\theta_{i}-\theta_{z}\right)\right] .
$$

Now consider the equilibrium and non-equilibrium contributions separately. After substituting equations (13), (14) and (15) into (48) a little algebraic evaluation leads us to the following relation:

$$
\sum_{\mathbf{x} \in A} \sum_{i} N_{i}^{(e)} U_{i}=\sum_{\mathbf{x} \in A} \rho \mathbf{u}^{2} \cos \left[2\left(\theta_{u}-\theta_{z}\right)\right]
$$


where $\theta_{u}$ is defined through

$$
\begin{aligned}
& u_{x}=\mathbf{u}^{2} \cos \theta_{u}(\mathbf{x}), \\
& u_{y}=\mathbf{u}^{2} \sin \theta_{u}(\mathbf{x}) .
\end{aligned}
$$

To find the non-equilibrium contribution we note that, at steady state, the evolution equation is reduced to

$$
N_{i}\left(\mathbf{x}+\mathbf{c}_{i}\right)=N_{i}(\mathbf{x})-\omega N_{i}^{(n e q)}+\Omega_{i}^{(2)}, \quad i=0, \ldots, 8
$$

where $\Omega_{i}^{(2)}=\left(\Omega_{i}^{r}\right)^{(2)}+\left(\Omega_{i}^{b}\right)^{(2)}$. For a lattice with well-defined boundary conditions

$$
\sum_{\mathbf{x}} N_{i}\left(\mathbf{x}+\mathbf{c}_{i}\right)=\sum_{\mathbf{x}} N_{i}(\mathbf{x})
$$

and therefore

$$
\sum_{\mathbf{x}} \sum_{i} N_{i}^{(n e q)} U_{i}=\frac{A_{r}+A_{b}}{2 \omega} \sum_{\mathbf{x}}|\mathbf{F}| \sum_{i}\left(W_{i} \frac{\left(\mathbf{c}_{i} \cdot \mathbf{F}\right)^{2}}{|\mathbf{F}|^{2}}-B_{i}\right) U_{i} .
$$

If we assume $\mathbf{F} /|\mathbf{F}|$ is constant we can write

$$
U_{i}=\left(\frac{\left(\mathbf{c}_{i} \cdot \mathbf{F}\right)^{2}}{|\mathbf{F}|^{2}}-\frac{\left(\mathbf{c}_{i} \cdot \mathbf{G}\right)^{2}}{|\mathbf{G}|^{2}}\right)
$$

where $\mathbf{G}$ is any vector perpendicular to $\mathbf{F}$. This then yields

$$
\sum_{\mathbf{x}} \sum_{i} N_{i}^{(n e q)} U_{i}=\frac{2\left(A_{r}+A_{b}\right)}{18 \omega} \sum_{\mathbf{x}}|\mathbf{F}| .
$$

Finally, combining the equilibrium and non-equilibrium contributions gives

$$
\sigma=\frac{\cos \theta_{z}}{M}\left(\sum_{\mathbf{x}} \rho \mathbf{u}^{2} \cos \left[2\left(\theta_{u}-\theta_{z}\right)\right]+\frac{2\left(A_{r}+A_{b}\right)}{18 \omega} \sum_{\mathbf{x}}|\mathbf{F}|\right) .
$$

The second term in equation (58) is relatively straightforward since $|\mathbf{F}|$ vanishes in non-interfacial regions but there appears to be no obvious general simplification of the first term (which is of second order in $\mathbf{u}$ ). It is, however, manageable in particular circumstances.

To first order in $\mathbf{u}$ we can neglect the first term in (58) to obtain

$$
\sigma=\frac{\cos \theta_{z}}{M}\left(\frac{2\left(A_{r}+A_{b}\right)}{18 \omega} \sum_{\mathbf{x}}|\mathbf{F}|\right) \sim \frac{A_{r}+A_{b}}{\omega},
$$

where $\omega$ determines the kinematic viscosity of the fluid through the relationship (77).

2.2.1. Plane interfaces Consider a thin plane interface parallel to the $y$-axis (such that $\cos \theta_{z}=1$ ) with colour symmetrically separated and assume there are no microcurrents $(\mathbf{u}=0)$, then the expression for surface tension (58) becomes

$$
\sigma=\frac{2\left(A_{r}+A_{b}\right)}{18 M \omega} \sum_{\mathbf{x}}|\mathbf{F}| .
$$

The stable interface cannot be centered on a single layer and as we integrate (43) along the line perpendicular to the interface three nodes will contribute to the surface tension, 
i.e there are three nodes with non-zero $\mathbf{F}$ : one lying on the line $x=x_{0}$ and one to both is left and right (Fig. 3). This corresponds to a $|\mathbf{F}|$ given by

$$
|\mathbf{F}|=C\left(\delta\left(x-x_{0}+1\right)+\delta\left(x-x_{0}\right)+\delta\left(x-x_{0}-1\right)\right),
$$

where $C$ is a constant and $\delta$ here refers to the Dirac delta function. Therefore

$$
\begin{aligned}
\sigma & =\frac{2\left(A_{r}+A_{b}\right) C}{18 M \omega} \sum_{\forall z, x}\left(\delta\left(x-x_{0}+1\right)+\delta\left(x-x_{0}\right)+\delta\left(x-x_{0}-1\right)\right), \\
& =\frac{\left(A_{r}+A_{b}\right) C}{3 \omega} .
\end{aligned}
$$

At steady state the colour gradient is constant, making it possible to estimate $|\mathbf{F}|$, and therefore $C$. By looking at the amount of red and blue particles at interfacial sites we find that $C=5 \rho / 2$ and therefore

$$
\sigma=\frac{5 \rho\left(A_{r}+A_{b}\right)}{6 \omega} \text {. }
$$

Figure 3. Symmetric separation about a thin interface. Circled nodes contribute to the theoretical expression for surface tension.

To verify the above relation a $64 \times 64$ square lattice domain was constructed with a vertical interface through the centre. Equal amounts of red and blue fluid filled the domain and, for simplicity, we set $\omega_{r}=\omega_{b}=1$ and $A_{r}=A_{b}=0.0001$. The system was allowed to evolve to a steady state before $P_{N}$ and $P_{T}$ were measured and the integral (43) approximated using a simple trapezoidal formula. Fig. 4 shows the numerical measurements of the mechanical definition $(+)$ and the theoretical prediction (solid line) of $\sigma$ as a function of density. The theory and numerics are seen to be in good agreement, confirming the validity of the above analysis.

We also test the capability of the model to predict Laplace's law for surface tension. We construct a $128 \times 128$ domain with a bubble of red fluid centered in the middle of the geometry and measure the pressure difference inside and outside the bubble. Laplace's formula is as follows:

$$
p_{i}-p_{o}=\frac{\sigma}{R}
$$

where $p_{i}$ and $p_{o}$ are the pressures inside and outside the bubble, respectively, $R$ is the radius and $\sigma$ the surface tension. Fig. 5 plots the pressure difference (calculated by 
Figure 4. The numerical measurements $(+)$ and theoretical predictions (solid line) of surface tension as a function of density.

equation (18)) against $1 / R$ using the following choice of parameters: $\rho_{r}=\rho_{b}=1$, $A_{r}=A_{b}=0.001 \alpha_{r}=0.1$. The numerical measurements are shown by the $\diamond$ symbols and the solid line is a linear fit passing through the origin, the slope of which gives the surface tension. The straight line is seen to be an excellent fit to the predicted pressure difference, thus demonstrating the ability of the model to predict surface tension.

Figure 5. The numerical measurements $\diamond$ and theoretical predictions (solid line) of Laplace's law for surface tension.

\subsection{Macroscopic Equations of Motion}

Although the addition of the two-phase component of the collision operator (23) enables the lattice Boltzmann model to simulate some multi-phase problems [19], the validity of the operator is not well understood. More specifically, its ability to handle flows with substantially different densities is untested and the theoretical considerations are incomplete. 
A collision operator of the form (23) or (26) aims at a discretisation of the term involving the distributed stress tensor because the colour gradient is an approximation of the derivative of a jump condition. However, it can be shown that equation (23) does not recover the correct form of the macroscopic force term after applying the ChapmanEnskog analysis. We will show, however, that a modification of the lattice Boltzmann collision operator can recover the correct form of the macroscopic equations. A single phase version of the Navier-Stokes equations are derived containing an appropriate source term, localized to the vicinity of the interface, to account for surface tension effects.

Since the two-phase collision operator $\left(\Omega_{i}^{k}\right)^{(2)}$ vanishes in regions containing just one fluid, the standard Chapman-Enskog analysis can be employed for each pure phase and the Navier-Stokes equations can be derived. At an interface the two-phase operator, $\left(\Omega_{i}^{k}\right)^{(2)}$ enters the analysis and a Taylor and Chapman-Enskog expansion of equation (2) yields, to first order in $\epsilon$ :

$$
\partial_{t_{1}} N_{i}^{(0)}+c_{i \alpha} \partial_{\alpha} N_{i}^{(0)}=-\omega N_{i}^{(1)}+\Omega_{i}^{(2)}
$$

where we have summed the 'red' and 'blue' contributions. We note that in the above $\omega=\omega(\phi)$ is the average relaxation parameter defined in equation (35). The mass and momentum constraints yield:

$$
\begin{aligned}
& \partial_{t_{1}} \rho+\partial_{\alpha} \rho u_{\alpha}=0 \\
& \partial_{t_{1}} \rho u_{\alpha}+\partial_{\alpha} \Pi_{\alpha \beta}=0
\end{aligned}
$$

respectively, where $\Pi_{\alpha \beta}$ is the momentum flux tensor, given by:

$$
\begin{aligned}
\Pi_{\alpha \beta} & =\sum_{i=0}^{8} N_{i}^{(e)} c_{i \alpha} c_{i \beta}, \\
& =p_{0} \delta_{\alpha \beta}+\rho u_{\alpha} u_{\beta},
\end{aligned}
$$

where the pressure $p_{0}=p_{0}^{r}+p_{0}^{b}$, is found from equation (18).

After the application of the mass constraint to the second order expansion of equation (2) we obtain:

$$
\partial_{t_{2}} \rho=0
$$

and combining this with the first order results shows that

$$
\partial_{t} \rho+\nabla \cdot \rho \mathbf{u}=0
$$

Application of the momentum constraint to the second order equation leads to the relation:

$$
\partial_{t_{2}} \rho u_{\alpha}+\partial_{\beta} Q_{\alpha \beta}+\frac{1}{2} \partial_{t_{1}} \partial_{\beta} \Pi_{\alpha \beta}+\frac{1}{2} \partial_{\beta} \partial_{\gamma} P_{\alpha \beta \gamma}=0
$$

where $P_{\alpha \beta \gamma}=\sum_{i} N_{i}^{(0)} c_{i \alpha} c_{i \beta} c_{i \gamma}$ and $Q_{\alpha \beta}=\sum_{i} N_{i}^{(1)} c_{i \alpha} c_{i \beta}$. The two-phase operator dictates the following form of the tensor $Q_{\alpha \beta}$ :

$$
Q_{\alpha \beta}=-\frac{1}{\omega}\left(\partial_{t_{1}} \Pi_{\alpha \beta}+\partial_{\gamma} P_{\alpha \beta \gamma}-\sum_{i} \Omega_{i}^{(2)} c_{i \alpha} c_{i \beta}\right),
$$


and therefore we find that

$$
\partial_{t_{2}} \rho u_{\alpha}+\partial_{\beta}\left(\frac{1}{2}-\frac{1}{\omega}\right)\left[\partial_{t_{1}} \Pi_{\alpha \beta}+\partial_{\gamma} P_{\alpha \beta \gamma}\right]+\partial_{\beta} \frac{1}{\omega} \sum_{i} \Omega_{i}^{(2)} c_{i \alpha} c_{i \beta}=0
$$

Now, adding equations (68) and (75) and using vector notation we find that

$$
\partial_{t} \rho \mathbf{u}+\nabla \cdot(\rho \mathbf{u u})=-\nabla p_{0} \mathbf{I}+\nu\left[\nabla^{2}(\rho \mathbf{u})+\nabla \nabla \cdot(\rho \mathbf{u})\right]-\nabla \cdot \mathbf{S},
$$

where

$$
\nu=\frac{1}{3}\left(\frac{1}{\omega}-\frac{1}{2}\right)
$$

is the kinematic viscosity and

$$
\begin{aligned}
S_{\alpha \beta} & =\frac{1}{\omega} \sum_{i} \Omega_{i}^{(2)} c_{i \alpha} c_{i \beta} \\
& =\frac{A|\mathbf{F}|}{\omega}\left(\frac{1}{|\mathbf{F}|^{2}} \sum_{i} W_{i}\left(\mathbf{c}_{i} \cdot \mathbf{F}\right)^{2} c_{i \alpha} c_{i \beta}-\sum_{i} B_{i} c_{i \alpha} c_{i \beta}\right) \\
& =\frac{A|\mathbf{F}|}{\omega}\left(\frac{1}{9|\mathbf{F}|^{2}} F_{\gamma} F_{\delta}\left(\delta_{\alpha \beta} \delta_{\gamma \delta}+\delta_{\alpha \gamma} \delta_{\beta \delta}+\delta_{\alpha \delta} \delta_{\beta \gamma}\right)-\frac{1}{3} \delta_{\alpha \beta}\right)
\end{aligned}
$$

which can be written as

$$
\mathbf{S}=\frac{2 A}{9 \omega|\mathbf{F}|}\left(\begin{array}{cc}
-F_{y}^{2} & F_{x} F_{y} \\
F_{x} F_{y} & -F_{x}^{2}
\end{array}\right) .
$$

The additional term in the Navier-Stokes equation viz. $\nabla \cdot \mathbf{S}$, arises from the effect of surface tension and can be expressed in terms of the fluid composition. The fluid composition is modelled using the colour difference, $\bar{\rho}$, which plays the role of an order parameter.

In the lattice Boltzmann model for immiscible fluids described here and elsewhere there are three fluid regions: homogeneous red and blue regions and a thin region near the interface where the two fluids mix. The method will not recover a sharp interface, i.e. one of zero thickness, but instead produce what is known as a 'diffuse' interface. Diffuse interface ideas were developed by Rayleigh [23] and by van der Waals [24], who proposed gradient theories for the interface based on the principles of thermodynamics. In diffuse interface models [25] a capillary stress tensor is used to model the interface between the two fluids. In this way a theory of the interface based on continuum mechanics may be developed and a modified Navier-Stokes equation can be derived with an additional term that accounts for surface tension (see Anderson et al [25], for example). The capillary tensor, $\boldsymbol{\Gamma}$, has the following form in terms of the colour difference:

$$
\boldsymbol{\Gamma} \sim|\nabla \bar{\rho}|^{2} \mathbf{I}-\nabla \bar{\rho} \otimes \nabla \bar{\rho} \simeq|\mathbf{F}|^{2} \mathbf{I}-\mathbf{F} \otimes \mathbf{F}
$$

where $\otimes$ denotes the outer product between two vectors.

Suppose that $\rho_{r}$ and $\rho_{b}$ are smooth functions that decay rapidly to zero in the interfacial region. Since $\bar{\rho}$ approximates a jump function, the surface delta distribution $\delta_{s}$, defined by

$$
\left\langle\delta_{s}, \phi\right\rangle=\int_{s} \phi d s
$$


where $s$ is the interface between the two phases, satisfies $\delta_{s} \approx C|\mathbf{F}|$ where $C$ is the inverse of the jump height. The surface distribution $\delta_{s}$ is only non-zero within a finite thickness transitional region near the interface since $\mathbf{F}=\mathbf{0}$ in pure phases. Therefore we can express the tensor $\mathbf{S}$ in the form

$$
\begin{aligned}
\mathbf{S} & =\frac{2 A}{9 \omega}\left(\mathbf{I}-\frac{\mathbf{F} \otimes \mathbf{F}}{|\mathbf{F}|^{2}}\right)|\mathbf{F}| \\
& =\frac{2 A}{9 \omega C}(\mathbf{I}-\mathbf{n} \otimes \mathbf{n}) \delta_{s}
\end{aligned}
$$

To first order in $\mathbf{u}, A / \omega$ is approximately proportional to $\sigma$, and therefore we have

$$
\mathbf{S}=B \sigma(\mathbf{I}-\mathbf{n} \otimes \mathbf{n}) \delta_{s}
$$

where $B$ is a constant. We note that

$$
\nabla \cdot(\mathbf{I}-\mathbf{n} \otimes \mathbf{n}) \delta_{s}=\kappa \mathbf{n} \delta_{s}
$$

where $\kappa$ is the mean radius of curvature of the interface defined by

$$
\kappa=-\nabla \cdot \mathbf{n}
$$

Note that the constant $B$ in (83) can be replaced with unity by scaling the two-phase collision operator (26) by an appropriate factor.

We remark that the collision operator defined in equation (23) would give the tensor $\mathbf{S}$ in the form

$$
\mathbf{S}=\frac{2 A}{\omega|\mathbf{F}|}\left(\begin{array}{cc}
2 F_{x}^{2}-|\mathbf{F}|^{2} & 4 F_{x} F_{y} \\
4 F_{x} F_{y} & 2 F_{y}^{2}-|\mathbf{F}|^{2}
\end{array}\right),
$$

which is not a correct discretisation of the term involving the distributed stress tensor in the Navier-Stokes equations.

\section{Numerical Simulations}

Consider two incompressible immiscible fluids moving in the $x$-direction under the influence of a horizontal pressure gradient $G$. If the flow is sufficiently small so that no instabilities occur with the interface remaining in the centre of the channel at all times then the analytical solutions for the steady flow are found to be

$$
\begin{aligned}
u^{r}= & \frac{G h^{2}}{2 \mu_{r}}\left[-\left(\frac{y}{h}\right)^{2}+\frac{y}{b}\left(\frac{\mu_{r}-\mu_{b}}{\mu_{r}+\mu_{b}}\right)\right. \\
& \left.+\frac{2 \mu_{r}}{\mu_{r}+\mu_{b}}\right], \quad-h \leq y \leq 0, \\
u^{b}= & \frac{G h^{2}}{2 \mu_{b}}\left[-\left(\frac{y}{h}\right)^{2}+\frac{y}{b}\left(\frac{\mu_{r}-\mu_{b}}{\mu_{r}+\mu_{b}}\right)\right. \\
& \left.+\frac{2 \mu_{b}}{\mu_{r}+\mu_{b}}\right], \quad 0 \leq y \leq h,
\end{aligned}
$$


Figure 6. Analytical (solid line) and numerical (+) measurements of velocity for two adjacent immiscible fluids (left plot) and three-layer Poiseuille flow (right plot).

where $h$ is the half channel width and $\mu_{r}$ and $\mu_{b}$ are the shear viscosities for red and blue fluids, respectively.

A horizontally periodic $128 \times 65$ lattice with a no-slip condition on the upper and lower walls was initialised with the upper half of the channel consisting of pure red fluid and the lower half pure blue. The centre line initially contained an equal number of each particle type. Both fluids were of unit density with $\omega_{b}=0.795229, \omega_{r}=0.360685$ (corresponding to viscosities $\mu_{b}=0.2525, \mu_{r}=0.75775$ ). We also set $\alpha_{r}=0.1, \delta=0.1$, $A_{r}=A_{b}=0$. Initially the system was at rest and a small force $G$ was used to mimic the pressure gradient and drive the flow based on the velocity $u_{I}$ at the center:

$$
u_{I}=\frac{g h^{2}}{\mu_{r}+\mu_{b}}=0.045
$$

Fig. 6 plots the analytical solution (solid line) and lattice Boltzmann prediction $(+)$ of the velocity profile. We see a very good agreement between the numerical and analytical predictions.

To demonstrate the role of the relaxation parameter, $\delta$, we perform the layered Poiseuille flow simulation as outlined above with $\delta=0.01,0.3,0.5,0.7,0.9$. Fig. 7 plots the velocity profile when $\delta=0.9$. Compared with the left-hand plot in Fig. 6, we see a smoother curve in the neighbourhood of the interface and a further departure from the analytical solution in this region. Table 1 shows the computed value of velocity at the points $y=-h / 2, y=0$ and $y=h / 2$ for each value of $\delta$ alongside the relative error between these and the analytical values. It is clear that as $\delta$ increases the relative error increases, which justifies our choice of $\delta=0.1$ in the simulations that follow.

Using the same value for the force $G$ we measure the velocity of a flow that has one fluid sandwiched between another less viscous fluid. Apart from the initial configuration this simulation is identical to the one described above. The analytical solutions to this flow are given by

$$
\begin{aligned}
& u^{b}=\frac{G}{8}\left(\frac{3 h^{2}}{\mu_{r}}+\frac{h^{2}-4 y^{2}}{\mu_{b}}\right), \quad-h \leq y \leq-\frac{h}{2}, \\
& u^{r}=\frac{G}{2 \mu_{r}}\left(h^{2}-y^{2}\right), \quad-\frac{h}{2} \leq y \leq \frac{h}{2},
\end{aligned}
$$


Figure 7. Analytic and numeric $(+)$ measurements of velocity of two adjacent immiscible fluids when $\delta=0.9$.

\begin{tabular}{|c|c|c|c|}
\hline$\delta$ & $u(-h / 2)$ & $u(0)$ & $u(h / 2)$ \\
\hline 0.01 & 0.03020 & 0.0451 & 0.04490 \\
error (\%) & 0.5 & 0.2 & 0.2 \\
\hline 0.1 & 0.03021 & 0.0451 & 0.04490 \\
error (\%) & 0.5 & 0.2 & 0.2 \\
\hline 0.3 & 0.0306 & 0.04520 & 0.0457 \\
error (\%) & 2 & 0.4 & 1.56 \\
\hline 0.5 & 0.0306 & 0.0454 & 0.0457 \\
error (\%) & 2 & 0.89 & 1.56 \\
\hline 0.7 & 0.0306 & 0.0458 & 0.0457 \\
error (\%) & 2 & 1.78 & 1.56 \\
\hline 0.9 & 0.0297 & 0.0459 & 0.0458 \\
error (\%) & 1 & 2 & 1.78 \\
\hline
\end{tabular}

Table 1. Comparison of velocity for two-layer Poiseuille flow using different values of $\delta$. The exact values are (to three significant figures): $u(-h / 2)=0.03 ; u(0)=u(h / 2)=$ 0.045 .

$$
u^{b}=\frac{G}{8}\left(\frac{3 h^{2}}{\mu_{r}}+\frac{h^{2}-4 y^{2}}{\mu_{b}}\right), \quad \frac{h}{2} \leq y \leq h
$$

and in Fig. 6 we plot the velocity predicted by the analysis (solid line) and the numerics $(+)$. Once again the results are in very good agreement.

The theoretical problem of finding the position of a fluid-fluid interface has nonunique solutions. However, experimental observations show immiscible fluids of the same density form spheres of one fluid within another. A stability analysis along with a study of the dynamics of rotating binary mixtures predicts the same phenomenon [1]. We test our model against these findings by adjusting the material parameters of the spinodal decomposition simulation discussed above. Starting with a random mixture of fluid in a fully periodic $64 \times 64$ domain with $\rho_{r}=\rho_{b}=1, \omega_{r}=0.360685, \omega_{b}=0.795229$ (corresponding to viscosities $\nu_{r}=0.7575, \nu_{b}=0.2525$ ), $A=0.0001$, and $\alpha_{r}=0.1$ we view the fluid configuration at various time steps. It is important to note that the 
Figure 8. Distribution of colour at times $t=1000$ (top left) $t=3000$ (top right), $t=15000$ (bottom left), and $t=30000$. The white fluid is the more viscous.

mass of each species did not change throughout this experiment. In Fig. 8 we see how a mixture of two liquids of different viscosities evolves into a stationary configuration with one large low viscous bubble immersed in a more viscous fluid. This result seems to agree with the ideas of Renardy and Joseph [1]:

Perhaps there is a selection mechanism based on the stability to large disturbances, in which the stable configuration is the one that minimizes the surface area. This type of criterion would lead to large bubbles, even one large bubble, rather than many small ones.

When a rod or cylindrical drop of one fluid is immersed in another, surface tension causes it to deform and capillary waves are induced that make the drops surface oscillate about its equilibrium shape. This behaviour can be observed in numerical calculations by surrounding an initially square droplet of one fluid with another and monitoring its response. To perform this test with our model we let a $32 \times 32$ square of red fluid centered in a $64 \times 64$ grid evolve in time. The parameters chosen for this flow were: $\rho_{r}=2, \rho_{b}=1, \nu_{r}=\nu_{b}=1, A_{r}=A_{b}=0.01, \alpha_{r}=0.5$. The initial configuration is shown in the top left plot of Fig. 9 and we take snapshots of the flow at different timesteps. After 140 time-steps surface tension has caused the corners (which are the areas of high curvature) to collapse, which in turn pushes the center of the vertices outward, resulting in diamond-like formation. Surface forces are then strong in these new areas of high curvature, thus sending the drop into oscillation. This behaviour is observed in Fig. 9 and which shows the red drop collapsing to a smoothed square $(t=300)$ before returning to a smoothed 'diamond' shape. The frequency of this oscillation decreases in time and by 800 time-steps the red fluid has found its equilibrium spherical shape. 
The final radius of the bubble is approximately 18.05, which shows its area is the same as the initial square configuration.

Figure 9. Oscillation and relaxation of an initially square droplet of higher density fluid to its equilibrium spherical shape.

For a large density ratio test we look at the coalescence of two identical circular droplets. Two red bubbles of radius $R=18.2$ and density $\rho_{r}=2.261$ are placed very close together in the center of a $100 \times 100$ computational domain. The surrounding fluid is blue with density $\rho_{b}=0.122$, giving a density ratio of $\gamma=18.5$. To ensure a stable interface we set $\alpha_{r}=0.95$ and the other parameters are: $\nu_{r}=\nu_{b}=1$ and $A_{r}=A_{b}=0.008$. The initial configuration is shown in the top left plot of Fig. 10 . As soon as the simulation starts inter-molecular forces cause the bubbles to coalesce and we see in Fig. 10 how the two droplets merge together. Like the non-equilibrium rod test tension forces send the surface into oscillation before it reaches its equilibrium. The symmetries about $x=n x / 2$ and $y=n y / 2$ are preserved and in agreement with other researchers' results [26]. The oscillation is clearly visible in Fig. 11 which plots the droplet radius at $y=n y / 2$ as a function of time $t$. It is important to note that the relation $R_{f}=\sqrt{2} R_{I}$, where $R_{f}$ is the final radius of the bubble and $R_{I}$ the initial radius, is satisfied.

\section{Conclusion}

In this paper a Rothman-Keller type lattice Boltzmann model has been developed for immiscible binary fluids using a D2Q9 lattice. An equilibrium function and collision operator for each phase has been derived, allowing each fluid to have to have its own density and viscosity while at the same time satisfying the necessary conservation laws and symmetry conditions. A theoretical expression for surface tension has been derived from this model and shown to be in good agreement with numerical measurements. The macroscopic governing equations are satisfied by the mesoscopic evolution of each phase. 
Figure 10. Plots showing the coalescence of two identical bubbles and its relaxation to an equilibrium configuration due forces at the interface.

Figure 11. The radius of the coalescing bubble at $y=n y / 2$ as a function of time. The solid line is a polynomial fit through the data points.

The model was first used to study Poiseuille flow in a two-dimensional channel for two and three layer configurations of immiscible fluids. Good agreement with the analytical solution was obtained in both cases. The three layer configuration maximises the mass flux for a given pressure gradient [1]. Using a body force of the same magnitude a greater maximum velocity is obtained than in the two layer configuration.

The thickness of the interface between the two fluids is controlled by the value of the free parameter, $\delta$. Sharp interfaces are achieved by choosing small values of $\delta$ while values close to unity lead to the interface being spread over several lattice cells. The results in this paper were generated using $\delta=0.1$. Larger values caused too much smearing of the interface and resulted in a less accurate prediction of the velocity profile near an interface. 
The model was then used to simulate the spinodal decomposition of a binary mixtures. In the case of a mixture of two fluids with the same density but different viscosities the model predicts that, in equilibrium, one large low viscous bubble is surrounded by the more viscous fluid. This prediction agrees with the analysis of Joseph and Renardy [1] who show, using rigorous mathematical arguments, that stable solutions to the equations for rigid motions of two liquids can be framed as a minimisation of energy problem, the only global solution to which is one large sphere. We have also performed simulations to demonstrate that this model can predict binary flows with much larger density ratios than other R-K type lattice Boltzmann methods.

\section{References}

[1] D.D Joseph and Y.Y Renardy. Fundamentals of Two-Fluid Dynamics. Springer-Verlag, New York, 1993.

[2] D.H Rothman and J.M Keller. Immiscible cellular-automaton fluids. J. Stat. Phys, 52:1119-1127, 1988.

[3] U. Frisch, B. Hasslacher, and Y. Pomeau. Lattice gas automata for the Navier-Stokes equations. Phys. Rev. Lett, 56:1505-1508, 1986.

[4] J.A. Somers and P.C. Rem. Analysis of surface tension in two-phase lattice gases. Physica D, 47:39-46, 1991.

[5] H. Chen and X. Shan. Lattice Boltzmann model for simulating flows with multiphase components. Phys. Rev. E, 47:1815-1819, 1993.

[6] G. McNamara and G. Zanetti. Use of the Boltzmann equation to simulate lattice gas automata. Phys. Rev. Lett, 761:2332-2335, 1988.

[7] Dazhi Yu, Renwei Mei, and Wei Shyy. A unified boundary treatment in lattice Boltzmann method. AIAA, 41ST:2003-953, 2003.

[8] J. Chin, E.S Boek, and P.V. Coveney. Lattice Boltzmann simulation of the flow of binary immiscible fluids using the Shan-Chen microscopic interaction model. Phil. Trans. R. Soc. Lond. A, 360:547-558, 2002.

[9] H. Chen and X. Shan. Simulation of nonideal gases and liquid-gas phase transitions by the lattice Boltzmann equation. Phys. Rev. E, 49:2941-2948, 1994.

[10] K. Sankaranarayanan and S. Sundaresan. Lift force in bubbly suspensions. Chem. Eng. Sci, $57: 3521-3542,2002$.

[11] R.R Nourgaliev, T.N. Dinh, T.G Theofanous, and D. Joseph. The lattice Boltzmann equation method: Theoretical interpretation, numerics and implications. Int. J. Multiphase Flow, 29:117169, 2003.

[12] L. Luo. Theory of the lattice Boltzmann method: Lattice Boltzmann models for nonideal gases. Phys. Rev. E, 62:4982-4996, 2000.

[13] M. Swift, W. Osbourne, and J. Yeomans. Lattice Boltzmann simulations for non-ideal fluids. Phys. Rev. Lett, 75:830-833, 1995.

[14] E. Orlandini, M. Swift, and J. Yeomans. A lattice Boltzmann model of binary fluid mixture. Europhys. Lett, 17:479-484, 1992.

[15] T. Inamuro, N. Konishi, and F. Ogino. A Galilean invariant model of the lattice Boltzmann method for multiphase fluid flows using free energy appraoch. Comp. Phys. Comm, 129:32-45, 2000.

[16] Aiguo Xu, G. Gonnella, and A. Lamura. Phase seperation of incompressible binary fluids with lattice Boltzmann methods. Physica A, 331:10-22, 2004.

[17] L. Luo. Unified theory of lattice Boltzmann models for nonideal gases. Phys. Rev. Lett, 81:1618$1621,1998$. 
[18] A. Gunstensen and D. Rothman. Lattice Boltzmann model of immiscible fluids. Phys. Rev. A, 43:4320-4327, 1991.

[19] D. Grunau, S. Chen, and K. Eggert. A lattice Boltzmann model for multiphase fluid flows. Phys. Fluids. A, 5:2557-2562, 1993.

[20] J. Tölke, M. Krafczyk, M. Schulz, and E. Rank. Lattice Boltzmann simulations of binary fluid flow through porous media. Phil. Trans. R. Soc. Lond. A, 360:535-545, 2002.

[21] M. Latva-Kokko and D.H. Rothman. Diffusion properties of gradient-based lattice Boltzmann models of immiscible fluids. Phys. Rev. E, 71:056702, 2005.

[22] I. Halliday, S. P. Thompson, and C. M Care. Macroscopic surface tension in a lattice BhatnagarGross-Krook model of two immiscible fluids. Phys. Rev. E, 57:514-523, 1998.

[23] Lord Rayleigh. On the theory of surface forces-ii. Compressible fluids. Phil. Mag., 30:209-220, 1892.

[24] J. D. van der Waals. The thermodynamic theory of capillarity under the hypothesis of a continuous density variation. Translation in J. Stat. Phys. 20 (1979), 197-244, by J. S. Rowlinson, 1893.

[25] D. M. Anderson, G. B. McFadden, and A. A. Wheeler. Diffuse-interface methods in fluid mechanics. Annu. Rev. Fluid. Mech., 30:139-165, 1998.

[26] P Yuan. Thermal lattice Boltzmann two-phase flow model for fluid dynamics. PhD thesis, University of Pittsburgh, 2005. 